\title{
LE ROMAN ET LA COMMUNE
}

\author{
JALONS POUR UNE DESCRIPTION*
}

Le roman sur la Commune, qu'il se propose d'exalter la Commune ou de la rabaisser, qu'il ait un but politique et pédagogique, ou également des ambitions esthétiques, n'en a pas moins une base commune à tous ses auteurs: la volonté de décrire «la réalité». La notion flaubertienneju «livre sur rien", l'idée qu'un texte naît et se développe selon des lois qui lui sont propres et qui n'ont rien de commun avec la "réalité", semblent complètement étrangères à nos romanciers. Certes, il leur arrive parfois de dépasser le naturalisme et le réalisme (par exemple, Cladel à la fin d'I.N.R.I., Zola à la fin de La Débâcle), mais cela reste au niveau de la pratique du texte: leur théorie du roman reste globalement prisonnière de la description du réel, avec toutes les conséquences formelles, mais aussi politiques, qu'entraîne ce choix.

Les écrivains favorables à la Commune, en règle générale, se défient des recherches formelles, qu'ils conçoivent comme sans rapport avec la "réalités, et donc ne pouvant parler au public populaire. Comme l'écrit Vallès à propos de la peinture:

"Aussi le peuple rôde-t-il ennuyé et dédaigneux à travers ce monde d'enluminures artificielles et qui, même avec la meilleure intention de l'auteur, ne réussissent pas à traduire la vie populaire ... [...] Si la peinture ne se retrempe pas dans le courant des idées nouvelles, gare à elle! Elle sera l'art en décadence dans la France en révolution.» ${ }^{1}$

Dans cette optique, l'œuvre doit être, par son contenu, le véhicule de l'idée révolutionnaire: quant à la forme, elle doit être "correcte» et "compréhensible", selon l'expression du poète communard Chatelain, elle peut même être «belle», ${ }^{2}$ mais l'auteur n'a surtout pas à se préoccuper d' "art pur".

* Cet article est la publication in extenso d'une communication résumée prononcée au Colloque universitaire de Paris sur la Commune, le 23 mai 1971.

1 Jules Vallès, in: Le Cri du Peuple, 3 mai 1884.

2 La préface de Lucien Descaves à I.N.R.I. de Léon Cladel (Paris, Librairie Valois, 1931) frappe par l'inconsciente tentative de récupération qui se manifeste dans les éloges adressés au style "ciselé», "buriné», de Cladel. 
Une telle attitude n'est pas sans soulever mainte difficulté. Tout d'abord, elle tient pour acquise la distinction entre fond et forme, dissociant un "fond» révolutionnaire et une "forme" héritière de la tradition, sans s'interroger sur la légitimité ni sur la possibilité de cette analyse. Que le roman anti-communard, réactionnaire sur le plan politique, le soit aussi dans le domaine de l'art, n'a rien pour surprendre ou pour inquiéter; plus inquiétant est de constater que le roman procommunard, qui se veut explicitement au service de la Révolution, n'en est pas moins, par son esthétique et les formes qui en découlent, un roman le plus souvent traditionnel, donc en contradiction grave avec les intentions de ses auteurs. A l'extrême limite,

"ces signes spécifiques, ces conventions de genres littéraires qui font de l'expression littéraire un discours n'ayant délibérément aucun rapport avec la réalité [...] lorsque les écrivains militants les utilisent $[\ldots]$ gardent le poids de tous les récits qu'ils ont construits et entraînent l'histoire réelle de la Commune dans le domaine de l'imaginaire. $\rangle^{1}$

Les questions qui surgissent alors peuvent, en un premier temps, et de façon certainement trop ambitieuse, se formuler ainsi:

- comment se manifeste, dans les romans favorables à la Commune, la dissociation entre une intention révolutionnaire et un texte traditionnel?

- quel rôle joue, dans cette dissociation qui se produit contre la volonté de l'auteur (et souvent à son insu), la préoccupation d'être compris d'un public populaire?

- cette carence des écrivains, même les plus favorables à la Commune, est-elle liée à leur statut social, à l'insuffisance de leur analyse politique, à une carence de l'événement même (la Commune envisagée sous l'angle du seul échec), à une sorte de «fatalité formelle», que nous venons d'évoquer, et à laquelle l'écrivain révolutionnaire ne pourrait échapper que par une totale destruction des formes (?) ? Et si l'on admet que ces diverses causes interviennent, comment les hiérarchiser?

Il n'est pas question ici de parvenir à résoudre tous ces problèmes, tant s'en faut. Nous proposons seulement, à l'aide de quelques analyses sur un ensemble de textes donné, de tâcher d'élucider le rapport complexe entre littérature et histoire, sur le point crucial de la Commune.

1 Annie Petit, Mouvements sociaux et phénomène littéraire au XIXe siècle en France: naissance d'une littérature ouvrière, Mémoire de maîtrise (dactylographié), Aix-en-Provence 1970, p. 117. 
Le premier problème qui se pose au roman sur la Commune est celui du roman historique. Comme l'a montré Lukacs, ${ }^{1}$ le roman historique présente un des cas particuliers de la crise qui affecte le réalisme bourgeois à partir de 1848. Jusqu'alors, les penseurs réactionnaires avaient fait preuve d'une certaine impartialité dans l'investigation, ils «[avaient] exprimé leurs idées encore assez librement, puisque les dangers sociaux auxquels ils faisaient allusion et qui déterminaient leurs pensées n'étaient pas encore devenus d'une actualité menaçante au point de susciter des falsifications apologétiques» ${ }^{2}$. Il n'en est plus de même après 1848 , et plus encore après $1870 .^{3}$ Balzac n'aura pas d'héritiers dans la génération des Maxime du Camp, des Coppée ou des Gautier. ${ }^{4}$ Les romans écrits contre la Commune abondent en "falsifications apologétiques"; la question se poserait de savoir si le même phénomène ne se produit pas dans les romans favorables à la Commune, en particulier dans ceux dont les auteurs n'ont pas participé à l'événement ou sont sociologiquement trop éloignés du peuple qu'ils entendent représenter.

Mais le roman sur la Commune est un roman historique d'un genre particulier. Il manque le plus souvent de recul temporel: contrairement à un Walter Scott, ou plus tard à un Zévaco, les romanciers sur la Commune prennent la plume peu d'années après l'événement. Or, si la Commune proprement dite est très limitée dans le temps, elle garde une actualité effective, en raison tout d'abord de la prolongation des procès, de la lutte pour l'amnistie qui se poursuit jusqu'en 1880, mais aussi et surtout parce que le souvenir de la Commune, qu'elle soit modèle ou traumatisme, reste longtemps vivant dans la conscience des Parisiens. Dans bien des cas, le roman apparaîtra donc comme une sorte de "Journal" de l'histoire, soit que cela affecte directement la forme de l'écriture (Mes Cahiers Ronges de Vuillaume), soit qu'il se donne comme une autobiographie lointaine ou non, déguisée ou non (Histoire d'un Trente-Sous de Sutter-Laumann, Un Exilé de Georges Renard...) soit enfin qu'il prenne l'allure d'une enquête ou d'une recherche du temps perdu (Philémon, Vieux de la Vieille de Lucien Descaves.)

Mais lorsqu'un Cassou ou un Chabrol écrivent un roman sur la

1 G. Lukacs, Le roman historique, trad. Sailley, Paris, Payot, 1965, pp. 190 sq.

2 Ibid., p. 194.

${ }^{3}$ L'ouvrage de Lukacs est malheureusement muet sur le point qui nous intéresse, passant des années 1850 à la période de 1914; il évoque pourtant des œuvres à rattacher à la littérature de la Commune, notamment Quatrevingt-Treize de Hugo.

4 Dans le cas de Gautier se vérifie particulièrement l'assertion de Lukacs, Gautier passant d'une attitude frondeuse et relativement "progressiste" dans les années 1830 à une totale "réaction" en 1871. 
Commune, il est clair que pour eux le problème de la transposition historique se pose; ${ }^{1}$ à la fois parce qu'eux-mêmes n'ont pas vécu l'événement, et surtout parce qu'à travers 1871 ils relatent les années 1930 , voire 1968. Les Massacres de Paris (Cassou) et Le Canon Fraternité (Chabrol) doivent donc faire l'objet d'une double lecture: en fonction de la chose racontée (la Commune) et en fonction de la situation historique de l'auteur (point de vue constamment souligné par Chabrol). Une double lecture du même ordre sera exigée par des textes comme Germinal ou Quatrevingt-Treize, romans qui apparemment ne parlent pas de la Commune, mais qui, écrits ou achevés après 1871, en sont tout imprégnés. Le rapport du roman à l'histoire se situe donc à plusieurs niveaux.

Il peut être de l'ordre de la négation: ce sera souvent le cas dans les romans hostiles à la Commune. L'histoire y devient "privée», elle n'est qu'un cadre, un décor, c'est-à-dire qu'elle n'est plus. Ce phénomène n'est pas apparu après 1871, il date des années 1850 , mais il s'aggrave significativement dans les cuvres d'après la Commune. Pour l'écrivain anti-communard, il s'agit de vider l'événement de son sens historique: l'histoire sera certes présente, on ne peut entièrement l'évacuer, mais ce sera une présence creuse, comme rongée: «On peut suivre pas à pas le rapport chronologique de l'action privée avec l'histoire [politique], mais rien que le rapport chronologique. Car ces guerres sont menées, ces paix sont conclues, sans que le lecteur y comprenne quoi que ce soit, sans même qu'il s'y intéresse. L'action centrale n'a rien à voir avec ces événements.» $\|^{2}$ Le roman anti-communard sera donc, le plus souvent, une histoire d'amour, dont les protagonistes appartiendront en général aux couches sociales supérieures: et comme l'histoire, le monde du travail en sera exclu, ou faussement représenté: «La vie professionnelle apparaît morte: tout ce qui est humain est complètement recouvert par les sables désertiques de la prose de la vie capitaliste. Les naturalistes postérieurs - même Zola - se précipitent sur cette prose et la placent au centre de la littérature, mais ils fixent et perpétuent seule-

1 Ajoutons prudemment, car ce n'est pas de notre compétence, le problème de la transposition linguistique, pour Chabrol surtout. La transcription écrite du parler de Marthe par exemple, avec ses "déformations" qui tentent de restituer un accent bellevillois en 1871, phénomène parlé et archaĩque, par un écrit de 1970, même en admettant une "stabilité" du parler populaire, risque de tomber dans la réfection linguistique la plus arbitraire: en fait, le modele n'est pas le parler de Marthe (personnage fictif) mais l'écrit des romanciers populistes. - Ce problème n'est pas propre à Chabrol: Vallès pratique par exemple une distanciation poétique; pour Descaves joue une distance sociale donnée, à laquelle il tente aussi de répondre par une poétique; mais le problème d'une langue archaïque ne se pose pas à eux.

2 Lukacs, op. cit., pp. 223-224. 
ment ses traits atrophiés, en limitant leur tableau à une description du milieu réifié. $)^{1}$

Un bon exemple de cette privatisation de l'histoire est fourni par l'œuvre de Coppée. La plus célèbre de ses nouvelles sur la Commune, dont le titre significatif, Une Idylle pendant le Siège, annonce assez l'orientation, narre les amours de Gabriel, un petit bourgeois à âme d'artiste, et d'Eugénie, une jeune femme parée de toutes les vertus: mariée certes, mais si mal! à un mauvais ouvrier qui deviendra Communard, de sorte qu'on ne peut guère reprocher son adultère à la malheureuse. ${ }^{2}$ Les événements de la guerre, de la défaite, de la proclamation de la: République..., ne servent que de toile de fond à l'intrigue amoureuse. Le récit est parlant de lui-même, mais Coppée prend soin de le souligner par de constants commentaires, redondants, mais intéressants par là même:

"On ne lui [à Gabriel] connaissait pas d'opinion politique.»"

"Ah! c'est vrai, la guerre! pensa-t-il, je l'avais oubliée.»"

«Gabriel était si loin de cet ordre d'idées qu'il ne comprit même pas ce que l'inquiétude de Cazaban avait de peu patriotique; ${ }^{5}$ seulement le mot du Méridional lui rappela la guerre. [...] < Tant pis pour ta République! [...] je suis sûr que nous serons vainqueurs sur toute la ligne.> $>\|^{6}$

La défaite de Wissembourg, que Gabriel apprend par l'effervescence de la rue, "dissipa d'abord sa langueur amoureuse». ${ }^{7}$ Trop d'indifférence à l'événement, en effet, risquerait d'aliéner au héros les sympathies du lecteur; aussi Coppée précise-t-il que Gabriel «n'était pas un égoïste; il aimait son pays tout comme un autre ${ }^{8}$ mais bien vite, Gabriel oublie le massacre pour ne songer qu'à son amour, et, par une figure hardie, tout le sang versé à Wissembourg se "miniaturise" jusqu'à devenir la goutte de sang qui perlait au doigt d'Eugénie quand elle s'est piquée en brodant. ${ }^{9}$ Ce genre de contrepoint, qui apparaît dans le récit pour la

1 Ibid., p. 230.

2 Nous évoquerons plus loin le personnage de Clément, le mari d'Eugénie, parmi les représentations hostiles du Communard.

${ }^{3}$ F. Coppée, Une Idylle pendant le Siège, éd. Lemerre des Euvres Complètes, tome III, p. 6.

4 Ibid., p. 11.

5 Cazaban, l'étudiant républicain, futur Communard, craint une victoire de "Badinguet" qui consoliderait sa dynastie...

6 F. Coppée, op. cit., p.15.

$\checkmark$ Ibid., p. 18.

8 Ibid., p. 18.

9 Ibid., pp. 18-19. 
première fois et de la façon la plus nette, sera constant par la suite.

Sedan, nous l'apprenons en même temps que Gabriel, de la bouche de sa mère qui l'a appris de la crémière! ${ }^{1}$ Dira-t-on que le cadre de la nouvelle est Paris et que Coppée n'avait pas à en sortir? Soit, mais les tableaux parisiens de l'insurrection nous laissent également désappointés: le 4 Septembre, Gabriel, tout absorbé par un rendez-vous manqué, ne voit rien de ce qui se déroule sous ses yeux, mais Coppée est là heureusement pour débrouiller le chaos et nous donner à voir la vérité: "Le spectacle grandiose promis ${ }^{2}[\ldots]$ n'était autre que celui d'une joie indécente chez un peuple que frappait un si effroyable désastre et que menaçait un si imminent péril. ॥* Engagé dans la garde nationale (c'est, il est vrai, dans un bataillon bien pensant), Gabriel n'y trouve qu'une occasion d'oisiveté pour rêver à son amour. Le spectacle des troupes vaincues et le sentiment de culpabilité qu'il éprouve à s'abstraire de la guerre l'amènent à se demander "s'il [est] un monstre. Le pauvre enfant n'était qu'un amoureux." "L'amour est supérieur à toutes les folies des hommes. $\|^{5}$ Coppée à la fois excuse son héros et aggrave l'espèce de schizophrénie qui le sépare de l'histoire: par un "jeu criminel de la mémoire», les "dates sanglantes» de la guerre ne servent qu'à rappeler à Gabriel les épisodes de son aventure amoureuse:

"Il leur fut un paradis, cet effroyable mois de janvier... $)^{6}$

"Ils furent heureux le soir du massacre de Buzenval: ils furent heureux le 22 janvier; ils furent heureux - ô honte! - le jour de la capitulation.

Mais, par un ordre étrange du hasard, ils le furent ce jour-là pour la dernière fois.n ${ }^{7}$

Les amants sont en effet séparés, d'abord par une maladie d'Eugénie qui ne se termine que le 17 mars..., ensuite par l'insurrection, car Gabriel suit à Versailles le ministère qui l'emploie comme gratte-papier; quand il rentre à Paris, peu après la Semaine sanglante, il n'y retrouve plus Eugénie, qui, son mari déporté, s'est réfugiée en province, il ne

1 Ibid., p. 27.

2 Par Cazaban qui entraîne Gabriel dans la foule en lui promettant «le grandiose spectacle de Paris délivré" (p. 31). Notons que Cazaban, tout futur Communard qu'il soit, est dupe du 4 Septembre, contrairement, par exemple, à Urbaine dans I.N.R.I.

3 F. Coppée, op. cit., p. 31.

4 Ibid., p. 37.

5 Ibid., p. 41.

6 Ibid., p. 45.

7 Ibid., p. 46. 
saura jamais où. C'est dire que la Commune n'apparaît, au premier abord, que comme un regrettable accident de parcours dans une "Idylle» à laquelle le lecteur est invité à accorder son adhésion.

Mais le récit est-il si innocent? Présenter la Commune sous ce jour, en faire le simple décor d'une intrigue bourgeoise, est un acte politique. Coppée ne se contente pas de peindre les Communards sous des traits ridicules ou odieux, ${ }^{1}$ c'est l'événement même qu'il tente de vider de son sens, déplorant la «folie» de la "guerre civile», qui se livre "sous les yeux de l'étranger", approuvant le patriotisme des gardes nationaux mais regrettant les excès auxquels il a abouti, ignorant le contenu social de la Commune.

Le même mécanisme se retrouve dans une nouvelle moins connue de Coppée, Toute une Jennesse: outre la peinture de toute sorte de fauteurs de troubles, comme le typographe Combarieu, ou de stratèges de café, comme les «Barbes», Coppée s'attache à mettre l'événement entre parenthèses. Il décrira longuement la bataille de Champigny, mais n'évoquera la Commune que dans un très bref monologue intérieur du héros, pour n'envisager d'ailleurs, de façon anecdotique, que la survie des Communards. Ainsi, dira-t-il, parmi les anciens du "café de Séville»,2

"On compte huit députés, trois ministres, deux ambassadeurs, un receveur général, et trente forçats qui attendent à Nouméa l'heure tardive de l'amnistie. ${ }^{3}$ Le plus intéressant, tout bien considéré, est encore ce sectaire imbécile, ce vieux fanatique de Dubief, ${ }^{4}$ celui qui ne buvait jamais que de l'eau sucrée; car lui, du moins, s'est fait tuer sur un tas de pavés par un feu de peloton des Versaillais. $\rangle^{5}$

Ce passage est caractéristique: il juxtapose un scepticisme de bon ton sur la destinée humaine (les uns deviennent ambassadeurs, les autres forçats) et une admiration humaniste pour l'adversaire quand celui-ci se fait tuer. ${ }^{6}$ Là encore, l'événement sort exsangue de la page. ${ }^{7}$

Mais l'œuvre de Coppée n'est nullement une exception: le même phénomène joue dans nombre de nouvelles ou de romans situés pen-

1 Citons les personnages de Cazaban, de Clément, de $\mathrm{M}^{\mathrm{me}}$ Henry, "Communarde" occasionnelle dont Coppée souligne l'opportunisme (cf. infra).

2 Le café de Madrid, l'un des lieux de réunion des républicains sous l'Empire.

3 Souligné par nous.

- Delescluze.

5 Toute une Jeunesse, op. cit., pp. 500-501.

- A comparer avec la critique de classe que Cladel, par la bouche d'Urbaine, fait du même Delescluze: à Râtâs qui, ému par le désespoir du vieux leader, veut lui tendre la main, Urbaine oppose l'incapacité de Delescluze et le caractère tardif et dérisoire de son "repentir".

$?$ D'autres nouvelles de Coppée illustrent la même technique: Euvres Posthumes, La Médaille, Le Naufrage de l'Inflexible, etc. 
dant la Commune: Les Désirs de Jean Servien (Anatole France), où la Révolution n'est que le cadre des rancœurs du héros, et ne procure à son amour déçu qu'une compensation burlesque avant de devenir l'instrument de sa mort: Thérèse (Hector Malot), où tout l'événement est vu au travers des mésaventures d'Edouard, où le lecteur ignore tout des raisons de la Commune, ignore sa chute, ignore la répression; Un Crime d'Amour (Paul Bourget), où 1871 n'est pour le héros qu'un souvenir d'enfance vécu naïvement et jamais interprété à la lumière du savoir de l'adulte; Césarine (Jean Richepin), Les Contes du Lundi (Alphonse Daudet), L'Apprentie ${ }^{1}$..., autant de textes qu'on serait tenté de dire "prétextes", oubliant qu'ils sont dictés par un choix politique d'autant plus efficace qu'il est masqué.

Dans les romans pro-communards au contraire, l'histoire doit «fonctionner", c'est-à-dire être la véritable trame des destins individuels: «les crises historiques présentées sont des éléments directs des destinées individuelles des personnages principaux et forment par conséquent une partie organique de l'action elle-même. [...] L'aspect individuel et l'aspect socio-historique sont inséparablement liés aussi bien en ce qui concerne la caractérisation que la conduite de l'action.." ${ }^{2}$ En fait, l'examen des textes montre qu'il s'agit là d'un idéal rarement réalisé. Par exemple dans La Colonne, il y a bien un lien entre le drame historique et le destin des personnages: mais n'est-ce pas parce que Descaves a isolé, dans l'ensemble de la Commune, un épisode significatif certes, mais nullement exhaustif? Aussi, à ce roman ambitieux, mais faussement parfait, préférerons-nous, comme Descaves lui-même, ${ }^{3}$ l'admirable Philémon, qui paradoxalement, avec des moyens réduits, ${ }^{4}$ fait vivre et revivre l'histoire: mais faut-il alors se résigner à n'écrire - et à ne lire - que "Vingt Ans Après»?

Ce regret, cette interrogation, c'est peut-être précisément ce qui fait le charme et l'épaisseur temporelle du récit de Descaves. L'approche de l'objet, dans sa lenteur même, est belle: Descaves ne feint pas de nous précipiter au milieu de l'événement communaliste, au contraire, il

1 Le cas de L'Apprentie (Gustave Geffroy) est plus délicat: cf. infra.

2 Lukacs, op. cit., p. 225.

${ }^{3} \mathrm{Au}$ romancier Antonin Lavergne, qui lui avait demandé La Colonne, il reprochait amicalement: "vous ne me demandez pas: Philémon Vieux de la Vieille pour lequel j'ai, moi, le plus de tendresse" (lettre inédite du 3 mars 1926, Archives Antonin Lavergne).

4 Moyens réduits parce qu'il s'agit d'une chronique intimiste, dont les personnages sont doublement des marginaux (par leur âge et par leur rôle modeste dans la Commune), et surtout par l'éloignement temporel, que Descaves souligne sans cesse au lieu de tenter de l'effacer comme le font la plupart des romanciers. 
calque, avec ses méandres et ses hésitations, la démarche du questionneur moderne en face d'un événement qu'il n'a pas vécu. Nous sommes tous l'auteur de Philémon: aux aguets comme lui, nous recueillons d'abord des indices incomplets, un bouquet d'immortelles, une visite à un cimetière, traces encore indéchiffrables qui ne prendront leur sens que plus tard, quand la forme, le "dessin dans le tapis», aura émergé. C'est alors seulement que Descaves fait intervenir explicitement l'histoire: mais celle-ci est présente d'emblée, dans la recherche même qui se livre au lecteur et se prend pour objet, sans le dire: il y a là une wabsence pleine» qui s'oppose à la présence creuse de l'histoire dans les textes anti-communards.

La mise en mots de l'histoire fera appel ensuite à toutes les ressources du récit: monologues d'Etienne (et les plus pathétiques sont sans doute ceux qu'il adresse à sa femme, frappée d'hémiplégie et devenue muette, pour tenter de susciter chez elle, par l'évocation de leur temps perdu, une étincelle de vie: symboliquement, discours de l'événement à l'histoire, discours qui se soupçonne inaudible et se poursuit pourtant), dialogues entre les autres "vieux de la vieille», chansons, vieux papiers, et nous ne résistons pas à l'envie de citer cet almanach que Descaves découvre chez ses voisins:

«[...] le jour de la fin mai, où le calendrier se couvrait de nuages. 20 mai. - Le râle de genêt arrive. La pivoine fleurit.

21. - Les Versaillais entrent dans Paris.

27. - Prise des Buttes-Chaumont. Le mûrier noir fleurit...»1

cette technique s'apparentant à celle du collage. Mais tous ces modes narratifs, qui jouent sur le souvenir et la distance, n'excluent pas pour autant une évocation directe: c'est ainsi que nous revivons, par les yeux d'Etienne, les dernières heures de la Commune, nous le voyons errer "comme un somnambule" ${ }^{2}$ dans Paris, brûler ses dernières cartouches Porte Saint-Denis, ou Porte Saint-Martin, il ne sait plus, mais, comme souvent, un détail absurde surnage: "un orgue de Barbarie nous jouait des motifs d'Orphée aux Enfers pour nous exciter». ${ }^{3}$ Mise en abyme hardie de l' "enfer" guilleret du Second Empire au milieu de l'apocalypse communaliste, mais qui nous convainc peut-être plus que si Etienne avait clamé du Pottier. La scène culmine en scène d'amour, lorsqu'Etienne retrouve Phonsine, la sœur d'un camarade de lutte, qui le cache: scène pudique, entre les lignes, mais dans le droit fil du récit,

1 Lucien Descaves, Philémon, Vieux de la Vieille, Paris, Librairie Paul Ollendorff, 1913, p. 200.

2 Ibid., p. 42 .

3 Ibid., id. 
comme les scènes équivalentes d'I.N.R.I. ou du Canon Fraternité, alors que chez Coppée ou Bourget l'amour est le contraire de la Commune.

Chez ceux-ci en effet, l'amour est l'anti-histoire, ce qui est malicieusement illustré par ce dialogue que Chabrol met dans la bouche de bons bourgeois:

"Qu'est-ce qui vous passionnait tant, que vous tourniez le dos à l'Histoire?

- Ce petit couple endormi là, sur la berge [...] Regarde s'ils sont mignons!" ${ }^{1}$

réplique qui déchaînera la fureur de Marthe. Au contraire, chez les romanciers pro-communards, l'amour et l'histoire se fondent:

«Je connais à présent le goût de la Révolution: c'est un air léger, doux à la peau, comme une peau fine et sombre, qui palpite lentement, marmonne et mordille la nuque." ${ }^{2}$

Cette fusion, ici vécue dans l'euphorie, pourra devenir déchirante, avec la séparation, l'exil, la mort (dans le cas de Philémon et Phonsine, la maladie qui fait d'elle une morte avant l'heure, puis sa mort qui laisse son époux sans ressources et l'accule au suicide): peu importe. L'essentiel est que l'histoire tisse le roman. Au temps discontinu, diaire, d'un Pipe-en-bois (Léon Deffoux), ou aux alternances narratives de La Commune (Paul et Victor Margueritte) ${ }^{3}$ s'oppose le Temps Retrouvé de Philémon, vision d'une histoire douée d'un sens, dans la double acception du terme.

Que l'histoire soit cadre ou fonction, cela ne résout pas entièrement pour le romancier le problème du déroulement du récit. Il ne s'agit évidemment pas ici de démontrer que le romancier, en tant que urapporteur d'histoire", oriente l'événement selon ses options personnelles, ce qui serait trop facile, mais d'essayer de repérer comment les modes mêmes du récit et le choix qui en est fait gauchissent l'événement. Or sur le plan romanesque, plusieurs questions se posent.

Tout d'abord, le récit des événements est fait par qui? Les romanciers sur la Commune adoptent trois solutions classiques, parfois de

1 J.-P. Chabrol, Le Canon Fraternité, Paris 1970, p. 94.

2 Ibid., p. 96.

3 Nous n'avons pu aborder ici le problème du temps du récit dans ses rapports avec l'histoire: il faudrait étudier notamment la question du décalage entre l'ordre chronologique des événements décrits et l'ordre de leur succession dans le texte. Cf. T. Todorov, Littérature et Signification, Larousse, 1967, p. 41. Les textes de Deffoux et des frères Margueritte seront analysés plus loin, mais sous un angle différent. 
façon homogène, parfois de façon mixte. Ou bien la narration est conduite par un romancier omniscient et omnipotent, qui sonde les cœurs d'autant de personnages qu'il en invente et nous fait assister à plus d'événements qu'un seul regard ne pourrait nous en livrer: c'est le cas de Coppée, de Zola... Ou bien le romancier part d'un "point de vue", utilisant la troisième personne du singulier mais nous donnant à voir l'histoire par les yeux de cette personne, qui devient ainsi une "première personne» masquée: c'est le cas d'Anatole France, de Geffroy... Ou enfin il choisit l'autobiographie (authentique ou en trompe-l'œil, peu importe ici), dans laquelle l'auteur et le narrateur se donnent comme confondus: c'est le cas de Vallès, de Vuillaume, du Descaves de Philémon, de Georges Renard, de Sutter-Laumann, etc. Ce dernier mode de récit, illustré par de grandes œuvres comme L'Insurgé ou Mes Cahiers Rouges, procure en théorie l'insertion maximale du roman dans l'histoire et de l'histoire dans le roman.

Encore faut-il, dans ce dernier cas, que le narrateur (et non l'auteur: nous réintroduisons ici la distinction qui se veut abolie dans l'autobiographie) se situe dans son texte, à tous les sens du mot. Un exemple montrera à quelle ambiguité aboutit le refus (volontaire ou non de la part de l'auteur) de situer le narrateur dans la narration: Pipe-en-bois témoin de la Commune, de Léon Deffoux. ${ }^{1}$ Le lecteur ne cerne que tardivement la raison de son malaise devant ce livre, dont les défauts apparents sont la superfluité (il ne relate rien qui ne soit bien connu, et ses "croquis sur le vif" eux-mêmes sont des stéréotypes), le pittoresque en stuc du style, mais qui semble se recommander par une réelle bienveillance envers la cause de la Commune. La défiance est progressivement éveillée par une phrase comme ce commentaire qui suit la fausse nouvelle de la mort de Vallès: "Je suis bien tranquille. Vallès, je l'ai vu partir, et il doit avoir quitté la France. $\rangle^{2}$ - phrase qui d'abord amuse par son caractère fabriqué (qui pouvait en juin 1871 se dire «bien tranquille» sur le sort de Vallès - sinon l'historien qui n'a qu'à regarder la date de sa vraie mort dans le dictionnaire?) et qui ensuite suscite la question: où donc était celui qui parle si paisiblement de la mort ou de la piteuse fuite des autres? Prêt à brûler ses dernières cartouches, caché, en route pour l'exil? Lorsqu'il se met enfin en scène comme "témoin détenu", la construction de la phrase elle-même est équivoque: "Les témoins détenus ont été introduits. Je suis avec eux.»" - car elle sépare «je» de (ils» et tend à rappeler tout en la cachant l'épithète infamante de «mouchard» appliquée à Pipe-en-bois. Le détachement

1 Paris, Les Editions de France, 1932.

2 Ibid., p. 207.

s Ibid., p. 227. 
olympien que le narrateur affecte tout au long du récit s'expliquerait par la certitude que lui-même a de se tirer d'affaire (et, dans le cas de l'auteur, Deffoux, par l'absence d'adhésion réelle à la cause communaliste ${ }^{1}$, , détachement plus irritant que celui par exemple des frères Margueritte, dans la mesure où ceux-ci ne prétendent pas offrir au lecteur l'authentique récit d'un "témoin". Au-delà du statut historique de Pipe-en-bois, qui nous importe en fin de compte assez peu, on voit que le texte de Deffoux est profondément équivoque: explicitement favorable aux Communards, malgré quelques critiques ou réserves, mais implicitement incertain, de par la situation flottante et louche du narrateur. C'est dire que le roman en première personne ne devra pas être interrogé seulement en fonction de ce qu'il dit, mais aussi et surtout en fonction du "comment il le dit", voire "comment il tait»."

De ce choix initial que fait le romancier, pouvons-nous déduire des catégories politiques? Il serait tentant d'établir des corrélations entre tel mode narratif et l'appartenance à tel ou tel camp: nous l'avons esquissé à propos de Pipe en-bois. Mais une systématisation serait prématurée: si de nos jours le choix du "romancier omniscient" apparaît souvent lié à une option non progressiste (encore est-ce loin d'être constamment vérifié), il serait historiquement inexact, à l'époque où nos romanciers écrivent, d'affirmer cette corrélation. Il nous faut pénétrer davantage dans le mouvement de constitution du récit, pour essayer de trouver des liaisons qui seront plus minces, portant sur des choix parfois de détail, mais peut-être aussi plus rigoureuses. Les principaux points envisagés porteront sur les passages obligés, l'effet de réel, les personnages et leurs fonctions. ${ }^{3}$

Le roman sur la Commune, comme tout récit, est constitué par une série de micro-récits, dont l'enchaînement se fait de façon assez peu variée, en règle générale. On constate la présence constante, dans la plupart de ces romans, de véritables "passages obligés», qui sont, par ordre chronologique: une défaite impériale, le Quatre Septembre, une manifestation pacifique, une réunion politique en milieu ouvrier, l'enlèvement des canons, un lynchage ou une exécution (le meurtre des

1 Cf. Eugène Schulkind, La littérature et la Commune de 1871, Thèse de doctorat d'Université, Paris 1951, p. 213 et passim.

${ }_{2}$ Une question à poser serait aussi: par quoi (et non plus par qui) est conduit le récit? ce qui entraînerait une étude des notions de "progrès" et de "fatalité", du rôle des masses, etc.

${ }^{3}$ Cette étude devra évidemment être poursuivie, tant pour approfondir les questions envisagées que pour en délimiter d'autres: le manque relatif d'études formelles sur notre type de roman, le caractère souvent inadéquat des catégories formelles du roman classique par rapport à notre sujet, ont été un handicap. 
généraux Lecomte et Thomas d'un côté, la mort de Flourens de l'autre; plus tard, la fusillade de la rue Haxo et la mort de Varlin et de Delescluze, sont les exemples les plus fréquents), l'arrivée d'une colonne de prisonniers à Versailles, les barricades, les incendies, la répression. Ces passages obligés, on le voit, dessinent une chronologie de la Commune. Mais on peut mettre doublement en question leur fidélité: sur le plan du contenu intrinsèque de tel ou tel passage, d'une part, sur le plan de leur enchaînement, d'autre part, car la chronologie avec ses apparences de rigueur peut bien n'être que «'histoire des sots», pour reprendre le mot de Balzac.

Or le contenu des passages est variable, et rassemble sous le même titre des développements assez divers. Prenons par exemple le meurtre des généraux Lecomte et Thomas, tel qu'il est décrit par les frères Margueritte, Cladel, Deffoux et Sutter-Laumann. ${ }^{1}$ Le lecteur constate d'abord l'inégal développement du même fait selon l'auteur, plus de dix pages dans I.N.R.I. et La Commune, moins de cinq pages dans Pipe-en-bois et L'Histoire d'un Trente-Sous. Cette inégalité quantitative est-elle politiquement signifiante? Sutter-Laumann et Deffoux, le premier ancien fédéré, le second simple "curieux", ont pour la Commune une sympathie romantique qui ne va pas jusqu'à l'adhésion aux positions politiques de la classe ouvrière, et les amène à faire un tri entre les actes de la Commune, notamment à déplorer le meurtre des généraux, que Sutter-Laumann n'explique que de façon anecdotique, ${ }^{2}$ et que Deffoux, plus justement, réfère à la haine qu'inspirait Clément Thomas comme fusilleur de $1848 .^{3} \mathrm{Il}$ est donc normal qu'ils tendent à escamoter le récit d'un geste qu'ils jugent regrettable, voire injustifiable. Mais les frères Margueritte, eux aussi horrifiés par ce meurtre qu'ils attribuent à la "tourbe», à l' "écume»" de Paris, le décrivent au contraire en détail, et le montrent en partie par les yeux de Du Breuil, l'officier versaillais et "républicain» qui est un des porte-parole des auteurs: donc la même cause (réprobation pour le meurtre) a produit des effets contraires. A l'opposé des uns et des autres, Cladel, le seul des quatre à justifier le meurtre de Thomas et Lecomte, développe sa fiction à la fois en longueur et, si l'on ose dire, en épaisseur romanesque, puisque c'est Urbaine elle-même qui fusille à bout portant Clément Thomas, reconnaissant en lui l'officier qui avait fait massacrer son père et sa mère en 1848. - Ce n'est donc pas le développement quantitatif du récit qui est

1 Nous nous limitons à ces quatre textes, mais le passage étudié apparaît en fait dans bien d'autres romans.

2 Cf. E. Schulkind, op. cit., p. 218.

s Pipe-en-bois, op. cit., p. 11 - "Ce sont d'anciennes balles de Juin.» (La Commune, op. cit., p. 83)

La Commune, op. cit., pp. 87 et 19. 
politiquement probant: ce qui l'est, c'est le jugement porté sur l'événement narré, jugement qui n'a pas à être explicite, puisqu'il peut s'exprimer par le recours au mythe, comme dans I.N.R.I.

Nous devons donc conclure que passage obligé n'est pas synonyme de stéréotype: certes, le stéréotype est fréquent, des deux côtés, mais il suffit qu'il puisse être dépassé par un auteur pour que la notion même de passage obligé cesse d'être dépréciative. Il en ira de même pour l'enchaînement de ces micro-récits: qu'il s'agisse d'un développement linéaire, comme c'est souvent le cas, ou d'un développement «à plusieurs dimensions", comme dans L'Insurgé ou Les Massacres, c'est la conception de l'histoire qu'a l'auteur, son insertion personnelle dans son temps, qui joue, et qui sécrète le stéréotype ou l'invention. Ajoutons ce qui peut-être paraîtra un paradoxe: à nos yeux, les romanciers communards n'ont pas l'exclusivité d'une vision riche de l'histoire, et donc du dépassement des stéréotypes. Si, comme l'a montré Paul Lidsky, une bonne part de la littérature anti-communarde est aujourd'hui caduque, certains de ses représentants ont su par le biais du mythe, même hostile, montrer la grandeur de la Commune. Deux exemples politiquement opposés illustreront cette idée: I.N.R.I. et Les Oiseaux... ${ }^{1}$, romans d'où nous extrayons deux scènes violemment antiréalistes, invraisemblables, choquantes, etc.: la crucifixion de Râtâs d'une part, l'orgie sanguinaire des Fédérés de l'autre. Aucun des auteurs ne prétend sérieusement nous faire croire à la véracité de leur récit: mais les deux scènes, par leur outrance même, sont significatives, non certes de leur "référent» (la répression versaillaise, la cruauté des Communards), lequel est secondaire, mais d'un référent plus vaste: l'affrontement entre "les deux Frances», son caractère vital. Ainsi la Commune, par Cladel comme par Bourges, se trouve prise au sérieux et retrouve un sens qui n'a rien à voir avec l'énormité de la chose représentée. Le mythe serait donc la parade au passage obligé, ce qui condamne le récit réaliste à une insuffisance radicale.

Dans le roman historique, dans le roman tout court, interviennent des personnages fictifs et des personnages historiques. Quelle place faire à ces derniers? En règle générale, ils jouent un rôle secondaire dans le récit, où ils n'apparaissent qu'en ombre portée:

"C'est précisément ce peu d'importance [dans le récit] qui confère au personnage historique son poids exact de réalité: ce peu est la mesure de l'authenticité: [ils] [...] sont introduits dans la fiction latéralement, obliquement, en passant, peints sur le décor, non détachés sur la scène: car si le personnage historique prenait

1 Elémir Bourges, Les Oiseaux s'envolent et les fleurs tombent, Paris 1893. 
son importance réelle, le discours serait obligé de le doter d'une contingence qui, paradoxalement, les déréaliserait [...] il faudrait les faire parler, et, comme des imposteurs, ils se démasqueraient. Au contraire, s'ils sont seulement mêlés à leurs voisins fictifs [...] leur modestie [...] met à égalité le roman et l'histoire [et] [...] donne au romanesque le lustre de la réalité, non celui de la gloire: ce sont des effets superlatifs de réel.»1

L'opinion qui prévaut généralement est que les romanciers ont raison d'éliminer ou d'estomper de leur récit les personnages historiques au profit des personnages fictifs, devenus plus (réels»: ce serait une marque de réalisme que de supprimer toute apparition des héros historiques pour ne montrer que des personnages obscurs, représentant le peuple. Tel est le sens de l'analyse d'Erckmann-Chatrian par Pissarev, critiquée par Lukacs ${ }^{2}$ : certes, Erckmann-Chatrian écrivent au sujet de la Révolution sans montrer Danton ni Robespierre, des guerres de 1'Empire sans montrer Napoléon: mais «est-ce réellement un avantage?» ${ }^{\mathbf{3}}$ On peut en douter: avec les apparences d'un réalisme minutieux, un tel roman ne livre au lecteur qu'un aspect du réel. Quelles seront les solutions adoptées par les romans sur la Commune? Elles sont essentiellement de deux types.

En premier lieu, le romancier peut choisir l'absence pure et simple du personnage historique: c'est le cas d'Une Idylle pendant le Siège, des Désirs de Jean Servien, des Contes du Lundi, des Oiseaux..., et, sur un plan légèrement différent, de L'Apprentie. Cette absence nous semble devoir être interprétée de deux façons: dans les premiers cas, où il s'agit de romans hostiles, elle est un aspect de "l'histoire privée», c'est-à-dire qu'elle tend à présenter la Commune comme une folie, une absurdité, bien secondaire par rapport aux "vrais problèmes», qui dans ces œuvres seront toujours anhistoriques. Cette perspective exclut l'apparition, même fugitive, d'un personnage historique dans une «histoire» qui tend à l'éliminer en même temps qu'elle élimine la véritable histoire.

Le cas de L'Apprentie est plus complexe. Dans ce roman relativement favorable à la cause communaliste, même s'il en montre la catastrophe, l'absence de personnages historiques, et la relative absence d' "histoire" tout court, pourraient être le reflet du pessimisme historique de Geffroy, ce qui nuance son adhésion: Geffroy "ressent l'écrasement de l'insurrection comme une fin, un anéantissement absolu du combat prolétarien. [...] C'est lui, probablement, qui se trouve le plus

1 Roland Barthes, S/Z, Ed. du Seuil, 1970, pp. 108-109.

2 Lukacs, op. cit., p. 235.

3 Ibid., id. 
près de l'état d'esprit populaire après le désastre que représente l'écrasement de la Commune." ${ }^{1}$ Mais ces absences sont surtout la marque d'un choix esthétique: la description à ras de terre du peuple parisien, qui n'approche jamais les grands, ce qui leur ôte tout droit à figurer dans le récit: le caractère «intimiste» et familial de la chronique crée une impossibilité supplémentaire à l'introduction du personnage historique.

L'absence totale est toutefois rare. En général, la solution qui prévaut consiste à montrer le personnage historique, mais de loin. Dans La Débâcle, par exemple, nous apercevons l'Empereur à plusieurs reprises, de loin, fantoche fardé et malade, ce qui symbolise sa condition d'individu dépassé, impuissant, jouet d'une épouse ambitieuse: explication réductrice de la guerre, mais nous n'en aurons pas d'autre, ou à peine! Les acteurs de la Commune ne nous seront pas montrés, ni leurs mobiles: Maurice et Jean seront seuls à représenter l'affrontement communaliste. ${ }^{2}$ Dans I.N.R.I., de tels effets de réel ${ }^{3}$ se réduisent à fort peu de chose sur l'ensemble du livre, et à l'exception de Delescluze, ${ }^{4}$ aucun personnage historique n'a de contact direct avec ses homologues fictifs. Dans le dialogue entre Urbaine et Delescluze se vérifie la "déréalisation" énoncée par Barthes: Urbaine et Râtâs sont plus "réels», ils pèsent le poids de notre sympathie et surtout de leur qualité de détenteurs du vrai politique. ${ }^{5}$

Sur ces deux solutions générales (absence ou vue de loin), chaque romancier greffe son choix particulier. Il serait fastidieux de mener la même analyse pour chacun d'entre eux: signalons les variations les plus caractéristiques.

Ecartons tout d'abord le déguisement du personnage sous une quelconque "clef" ("Polanceau" ou "Dubief» chez Coppée, "Tudesco" chez France, etc.) qui n'est qu'une sous-catégorie de la vue de loin. Plus intéressante est la solution alternée adoptée par exemple par les frères Margueritte: alternance de chapitres «historiques», où fourmillent les personnages historiques, et de chapitres romanesques, d'où ils disparaissent. Malgré son apparente richesse, ce n'est peut-être que l'aveu d'une

1 Palmiro Armando, Etude des constantes dans quelques romans versaillais et communards, Diplôme d'Etudes Supérieures (dactylographié), Aix-en-Provence 1970 , p. 61.

2 On sait avec quelle fidélité...

3 L'enterrement de Charles Hugo (pp. 151-152), la mort de Thomas et de Lecomte (161-169), la mort de Flourens (200-201), Wroblewski (242-243), Delescluze (215-224 et 248-250).

4 Et de Thomas: cf. supra.

s Mais dans ce cas précis, aucun des personnages en jeu, qu'il soit fictif ou historique, n'est doué de contingence : la déréalisation fonctionne au plan politique, non au plan narratif, nous semble-t-il. 
impuissance à faire la synthèse. La même alternance se retrouve, modulée, chez Chabrol, quand Flourens par exemple nous est présenté à la fois comme personnage de roman ${ }^{1}$ et comme extrait du Larousse: "Fils d'un physiologiste réputé, Gustave Flourens, etc.», ${ }^{2}$ ce passage étant en italique ${ }^{3}$ : l'italique bouche les trous historiques du récit ou sert de dictionnaire. Enfin, parfois, le personnage historique tend à se métamorphoser en personnage de roman: c'est le cas de Rossel chez Cassou. Beaucoup de romanciers ont été séduits par la figure de Rossel, mais ont commis l'erreur de lui faire écrire ou prononcer des phrases que le Rossel historique avait écrites ou prononcées: Cassou, en lui inventant un langage, fait de lui plus qu'un effet de réel, un personnage de roman à part entière. Aussi bien, quittant le personnage historique, est-ce au personnage fictif que nous allons maintenant nous attaquer.

Le rôle littéraire des personnages, dans le roman sur la Commune, est étroitement fonction de la conception que l'écrivain se fait du rôle de l'individu en histoire. Les romanciers anti-communards, pour minimiser la signification de l'événement, y verront non l'action du peuple entier, mais les menées d'une poignée d'agitateurs, souvent étrangers, toujours obscurs, manipulant un peuple amorphe et alcoolique d'où se détachent des Arthur, des Clément ou des Chouteau. Un tel tableau est dans l'ordre de leurs options politiques, et on ne peut s'en étonner. ${ }^{4}$ Mais en face de cette description, que proposent les romanciers procommunards? Le plus souvent, une galerie de portraits inverse mais équivalente. Peindre Rabouille ou Râtâs sous les traits les plus sympathiques est aussi mystificateur (peut-être plus) que le contraire. Car les uns et les autres renvoient à l'absence du peuple dans le roman: c'est l'impossibilité théorique d'apercevoir le vrai rôle du prolétariat et de le décrire, chez les romanciers hostiles comme chez les romanciers favorables, qui cristallise en personnages.

Cette vision partielle ou fausse du rôle de l'individu dicte à son tour des normes littéraires d'où le romancier favorable à la Commune peut rarement se dégager, en admettant qu'il le veuille: à cause de son public, qui exigera, croit-il, des "héros», ou parce que lui-même est attaché à ses formes littéraires et n'en conçoit pas d'autres, ou enfin faute de prendre conscience de son assujettissement à ces formes. $L e$ Canon Fraternité, par exemple, est-il un roman de 1970? On peut en douter. En dépit de ses habiletés contrapuntiques, on reste dans le grand roman de tradition balzacienne. Un siècle de recherches romanes-

1 Canon Fraternité, op. cit., pp. 104-108 et passim.

2 Ibid., pp. 104-105.

s L'italique, on le sait, représente ce que Florent écrit en 1914, lorsque, sexagénaire, il relit ses cahiers du temps de la Commune.

4 Cf. E. Schulkind, op. cit., pp. 215-220. 
ques n'a pas eu lieu. - En envisageant donc les principaux types décrits par le roman anti ou pro-communard, il faudra surtout souligner que la solution par personnages est une sorte de pis-aller. Certes, cette position est théorique, et elle fait bon marché de toute la tradition du roman occidental, qui est un roman à personnages: mais on ne peut non plus méconnaître la mise en question moderne du statut du personnage, et s'il est assez vain de regretter qu'un romancier de 1880 ou de 1900 n'ait pu la pressentir, on est peut-être en droit de demander à un romancier contemporain de ne pas complètement l'ignorer. S'il paraît excessif de dire, dans le contexte du roman français, que «l'histoire comme système de motifs peut entièrement se passer du héros et de ses traits caractéristiques", ${ }^{1}$ l'excès inverse est peut-être aussi grave, et contribue à ancrer chez le lecteur l'image tronquée d'une histoire à nouveau "privée".

Envisageons tout d'abord le roman anti-communard. Un inventaire quantitatif des personnages hostiles ou favorables à la Commune dans ces romans amène à une constatation paradoxale au premier abord: la liste des personnages de Communards ou assimilés est plus fournie que la liste symétrique. Cette remarque a été faite également par Paul Lidsky: "on trouve très peu de héros positifs pour symboliser l'autre camp", ${ }^{2}$ mais il n'en a pas tiré de conclusion. Or que peut signifier cette prolifération de personnages favorables à la Commune, c'est-à-dire porteurs d'une idéologie opposée à celle des auteurs? Elle a précisément pour fonction d'occulter l'idéologie. On décrira nombre de Communards (parfois, on se donnera le luxe de découvrir parmi eux des "égarés», voire des "victimes»), mais il ne sera pas nécessaire de décrire leurs adversaires, dans la mesure où leurs adversaires, c'est "tout le monde", tous les gens de bon sens, tous les gens de bien. Cette vaste catégorie se cristallisera en quelques héros positifs, représentatifs, mais elle ne peut être fournie, sous peine de se trahir. Le point de vue versaillais formant une sorte de basse continue à tous ces romans, ce serait, dans leur propre optique, une erreur esthétique et tactique que de fragmenter ce point de vue en trop de personnages. ${ }^{3}$

Cela dit, essayons de déceler les traits dominants des héros hostiles à la Commune dans le roman anti-communard. Ce héros est d'abord, sociologiquement, :plastique. Il est «au-dessus» des classes (attitude

1 Tomachevski, cité par Todorov, op. cit., p. 58.

2 Paul Lidsky, Les écrivains contre la Commune, Paris, Maspero, 1970, p. 116.

3 Ce serait une erreur d'entendre cela comme un machiavélisme conscient de la part de l'écrivain: il ne décide pas d'occulter l'idéologie (on ne peut que décider de la démasquer), mais son choix politique, même informulé, même inaperçu de lui-même, dicte le choix esthétique, comme nous en avons vu plusieurs exemples. 
défensive, corollaire de l'affirmation de classe des Communards) : qu'il soit paysan comme Jean Macquart, ${ }^{1}$ petit bourgeois prolétarisé mais sans conscience prolétarienne comme Gabriel Fontaine, ${ }^{2}$ bourgeois aisé mais artiste (donc plus tout à fait bourgeois...) comme Martial Poncet $^{3}$ ou Amédée Violette, ${ }^{4}$ aristocrate comme Paul de Ricieux ${ }^{5}$ ou M. de Grandpré ${ }^{\boldsymbol{6}}$ ou républicain comme Antoine Chamberlain, ${ }^{7}$ militaire de carrière comme Du Breuil, d'Avol ${ }^{8}$ ou Henryet, ${ }^{9}$ riche comme Edouard Chamberlain ${ }^{7}$ ou pauvre comme Jean Servien, peu importe: il n'est jamais défini d'abord par sa classe, mais par son appartenance à une réalité à la fois temporelle et spirituelle, qui trouve sa meilleure expression dans le personnage de Jean Macquart. Cette réalité, c'est la France de la religion, du travail, de la propriété: c'est Versailles. C'est pour défendre tout ce patrimoine moral contre les enragés du "parti du ventre" que cette coalition hétéroclite va prendre les armes.

Prendre les armes, métaphoriquement, s'entend. Certes, un d'Avol, un Henryet devront verser le sang: encore n'est-ce pas toujours sans répugnance. Mais les autres héros positifs du roman anti-communard luttent d'autre manière. Gabriel ou Amédée, qui traversent la Commune comme un mauvais rêve, Jean Servien, victime innocente d'une virago enragée, tous ont une fonction plus subtile: ils militent en sourdine, au second degré, par leur désintérêt pour la "politique»: «on ne [leur] connaît pas d'opinion politique», pour reprendre la phrase de Coppée sur Gabriel, mais leur dégoût pour la «barbarie» de la Commune est significatif. Sylphes du point de vue social, ils unissent la haine de l'artiste pour le "Bourgeois» philistin à une affection moins avouée mais plus profonde pour le même bourgeois sauveur de l'Ordre. - Certes, à entrer dans le détail, des différences plus ou moins grandes apparaissent: ainsi dans le roman des Margueritte, Du Breuil et d'Avol, tous deux nobles, tous deux militaires de carrière, n'en sont pas pour autant interchangeables. D'Avol, déterminé d'emblée contre la Commune, sans scrupules sur les modalités de la guerre civile, se heurte de plus en plus au cours du roman à Du Breuil, son ancien ami et compagnon d'armes, qui reste certes dans le camp versaillais mais est déchiré par cette guerre fratricide: il est visible que ce personnage

1 La Débâcle (Zola).

2 Une Idylle pendant le Siège (Coppée).

3 La Commune (frères Margueritte).

- Toute une Jeunesse (Coppée).

5 Césarine (Richepin).

- La Commune.

7 Thérèse (Malot).

8 La Commune.

- Le Chien perdu et la Femme fusillée (Arsène Houssaye). 
"conciliateur" a toute la sympathie des auteurs, qui le tuent à temps pour l'empêcher de participer à la répression et de ternir ainsi son auréole. - Mais ces différences, souvent négligeables quant à leur contenu, contribuent elles aussi à consolider l'idéologie versaillaise en feignant de la détruire: un camp où de si nobles affrontements ont lieu, où il y a tant de «différences» entre les êtres, est à la fois celui de la nuance tolérante et de la variété, preuve qu'il peut prétendre à être "le genre humain». Les porteurs de cette idéologie pourront même être anonymes: c'est le cas du Marin d'Arsène Houssaye, ou des marins "aux yeux clairs», respectueux et décidés, qu'envie le Communard déporté de Monologue à bord. ${ }^{1}$ Mais, anonymes ou héros, ils ont en commun leur fonction constructive: le meilleur exemple en est Jean Macquart, dont Zola fait explicitement le bâtisseur de l'avenir et le destructeur de la "pourriture" communaliste en la personne de Maurice. $^{2}$

Examinons à présent les personnages favorables à la Commune. Ils sont, nous l'avons dit, beaucoup plus nombreux. Leur classement peut s'opérer selon les critères suivants, qui se dégagent des romans euxmêmes: origine sociale du personnage, ses motivations pour être ou devenir Communard, son statut par rapport à la Commune (victime, dupe ou profiteur) et enfin son évolution, s'il y a lieu (c'est-à-dire son reniement). Le lien entre ces quatre critères est évident.

L'ouvrier d'abord. ${ }^{3}$ Cet ouvrier est rarement un prolétaire au sens moderne du terme. C'est généralement un artisan, il travaille «à son compte», et selon qu'il gagne bien ou non sa vie, il sera Communard d'entrée de jeu ou à l'issue d'une "conversion". Parmi les Communards de la première heure, citons le peintre en bâtiment Berru, ${ }^{4}$ le typographe Combarieu, ${ }^{5}$ l'entrepreneur en maçonnerie Clément, ${ }^{6}$ le peintre en bâtiment Chouteau, ${ }^{7}$ Fernol le charpentier. ${ }^{8}$ Les héros de Zola ayant été bien décrits, ${ }^{9}$ disons quelques mots des personnages moins connus. Le typographe Combarieu nous est décrit d'abord sous l'Empire: bien entendu, il conspire au lieu de travailler (il est vrai que c'est peut-être plus par désespoir que par conviction: sa femme l'a quitté...),

1 Dans Daudet, Contes du Lundi.

2 La Débâcle, chap. VIII, passim.

a Nous renvoyons ici à l'ouvrage cité de Paul Lidsky, notamment «Le jeune déclassé communard" et "Le mauvais ouvrier», pp. 98-108.

4 Jacques Damour, dans Zola, Naïs Micoulin.

5 Toute une Jeunesse.

- Une Idylle pendant le Siège.

7 La Débâcle.

8 La Commune.

P. Lidsky, op. cit., pp. 103-108. 
délaissant sa petite fille Rosine. Son physique n'est guère engageant: "Très brun, le teint livide, tout en cheveux et en barbe, et tâchant de se <aire la tête> de Jésus-Christ, l'ouvrier, vêtu de sa longue blouse noire de typographe, réalisait parfaitement le type du tribun de club, du <sublime> d'atelier. Franc-maçon probable, ivrogne solennel qui se serait pourtant grisé plus encore de grands mots que de petit bleu [...] toute sa personne faisait songer à un apôtre pochard.»1

Malgré ce physique christique, Combarieu est un buveur de sang, qui évoque "ce que nous avons vu aux journées de Juin et au Deux Décembre ${ }^{2}$ pour en tirer argument contre le socialisme humanitaire et réclamer le retour "aux grands moyens de 93 ". $^{2}$ Mais ces proclamations sanguinaires ne seront pas suivies d'effet: le personnage, compromis, disparaît. Après la Commune, le héros retrouvera la fille de Combarieu: elle a dix-huit ans et se prostitue. Elle explique à Amédée:

«papa ne s'occupait que de sa politique. A quinze ans, il m'a mise en apprentissage chez une fleuriste, et c'est l'amant de la patronne qui m'a débauchée.... [...]

[papa] est gérant d'un journal républicain, et n'a rien à faire que des mois de prison.... ${ }^{3}$

Ce bureaucrate de la Révolution ne s'est donc pas trop mal tiré d'affaire, mais sa fille, elle, paie cher les carences de l'éducation familiale. De la même façon, c'est parce que son mari, Clément, fume, boit et discourt dans les cafés, négligeant son entreprise qui périclite, qu'Eugénie, vertueuse, devient adultère. ${ }^{4}$ Quant à Fernol, plus bête que méchant, fort en gueule et Communard d'opérette, il est si peu convaincu de la justesse de sa cause qu'il s'échappera piteusement, déguisé en prêtre. ${ }^{5}$

Quant aux Communards convertis, ou pervertis, ils sont moins coupables que les premiers, qui les ont souvent entraînés: et la fin du texte les voit fréquemment saisis de remords et reconquis par la bonne cause, tels le ciseleur d'Arsène Houssaye ou l'exilé de Monologue à bord. Jacques Damour, qui ne reniera pas son adhésion à la Commune et sera

1 F. Coppée, Toute une Jeunesse, Lemerre, tome III, p. 401.

2 Ibid., id.

3 Ibid. p. 450. Notons que Coppée ne dit jamais que Combarieu participe à la Commune: nous avons vu comment, dans cette nouvelle, il réduisait l'événement proprement dit à un paragraphe. Mais Combarieu, en tant que "mauvais ouvrier" et conspirateur contre l'Empire, est typique comme représentation du Communard dans le roman hostile.

4 Une Idylle pendant le Siège.

5 La Commune. 
condamné à la déportation, est malgré cela moins blâmable que Berru, qui l'a perverti et qui se tirera d'affaire sans dommage.

La compagne de ces ouvriers est rarement une compagne de lutte. $\mathrm{Si}$ on excepte Thérèse et Rose dans La Commune, les mauvais ouvriers communards sont souvent flanqués d'une épouse qui réprouve secrètement ou ouvertement leur engagement politique, non qu'elle-même ait des idées précises dans ce domaine, mais elle sait, par intuition féminine, que la politique est toujours dangereuse pour son foyer:

«Et son imbécile de père qui s'amusait tout de même à mettre ses drapeaux, ses ballons rouges, et sa petite République de plâtre sur notre croisée!... M'a-t-il assez agacée!... Enfin, ces hommes, faut toujours que ça pense à la politique... Leur joujou, quoi?» ${ }^{1}$

Les femmes ou filles d'ouvriers seront donc hostiles à la Commune (Eugénie, Félicie Damour), hostilité souvent tacite, ou simplement étrangères (Céline de La Colonne, Fanchette dans Le Mur de Maurice Montégut, la sœur aînée de Cécile dans L'Apprentie, etc.). La Communarde du roman anti-communard sera rarement une ouvrière: elle appartiendra à une catégorie polymorphe, qui va de la fille à soldats à la demi-mondaine, mais qui exclut généralement la travailleuse.

Après les ouvriers et les ouvrières, le bourgeois rallié à la Commune. Il y en a de deux sortes dans le roman anti-communard: fonctionnaires gagne-petit comme Catisse, ${ }^{2}$ qui sont restés à Paris faute d'argent pour en partir et sont fidèles à la Commune parce qu'elle représente à leurs yeux «le gouvernement" et qu'elle leur permet de nourrir leur famille. Catisse sera fusillé par les Versaillais, mais ce n'était pas un insurgé. Mais il $\mathrm{y}$ a aussi et surtout le jeune bourgeois idéaliste ou bohême, étudiant ou ancien étudiant, artiste, qui a adhéré à la Commune par générosité ou rancœur. Cette catégorie est fournie: on pense tout de suite à Jean Servien, à Maurice de La Débâcle, ${ }^{3}$ mais ils sont les frères d'Adolphe Ducharme, ${ }^{4}$ de Thérould, Lévidov et Dury, ${ }^{\mathbf{5}}$ de Marius Cazaban, ${ }^{6}$ de Ludovic Charmes, ${ }^{7}$ de Fresnelay ${ }^{8}$ ou d'Agricol Mallet. ${ }^{9}$ Ils ont eu vingt ans sous l'Empire, ont trempé dans des conspirations

1 F. Coppée, La Médaille. Une femme d'ouvrier évoque la maladie qui a saisi son enfant un jour de 14 juillet, et s'irrite de voir son mari "pavoiser" au lieu de prendre part à son inquiétude (Lemerre, tome III, p. 241) .

2 La Commune.

3 Cf. P. Lidsky, op. cit., pp. 98-103.

- Le Chien Perdu...

5 La Commune.

- Une Idylle...

7 Le Mur.

8 Nos actes nous suivent (Paul Bourget).

9 Coppée, Euvres Posthumes. 
de café, comme Lemétreur, Plock ou «les Barbes», ${ }^{1}$ et ont vu dans la Commune une occasion de continuer leur petite guérilla urbaine et de retarder leur entrée dans le sérieux, dans la vie adulte. Certes, tous ces jeunes bourgeois ne sont pas identiques: certains sont des socialistes convaincus, des figures évangéliques qui ont mal trouvé leur point d'application (Agricol Mallet, Ludovic Charmes, Dury), d'autres sont des nihilistes (Lévidov, Maurice), d'autres enfin des opportunistes qui cherchent à profiter des malheurs de la patrie et se tireront toujours d'affaire, comme l'affreux Cazaban. Mais ils ont en commun d'être des marginaux, sans compétence sociale, des discoureurs: aucun bourgeois à part entière n'est représenté comme ayant adhéré à la Commune, et pour cause. Le roman anti-communard ne peint donc que de faux bourgeois dans le camp communard: la falsification de base atteint ici involontairement une réalité. ${ }^{2}$

La Communarde enfin. Nous avons dit qu'elle était rarement une ouvrière ou une femme d'ouvrier. Les nombreux personnages féminins du roman anti-communard renvoient en général aux stéréotypes de la presse versaillaise, puis plus tard de l'historiographie versaillaise; le roman n'engendre donc pas de types nouveaux. La cantinière qui exécute Jean Servien, les ménades cannibales d'Elémir Bourges, la fille demi-nue qui anime la "danse de Caraïbes» autour des cadavres de Clément Thomas et de Lecomte, ${ }^{3}$ la rousse dont l'obscénité agresse $\mathrm{Du}$ Breuil, ${ }^{4}$ la cantinière allumeuse du Concert de la Huitième, ${ }^{5}$ autant de figures anonymes et d'autant plus percutantes qui dessinent un portrait de la Communarde. Quand ce portrait se fragmente en personnages précis, il est à remarquer qu'il s'atténue. Angélique du Mur, Angélique du Chien Perdu..., Mme Henry ou Maddalena ${ }^{6}$ sont moins atroces, moins effrayantes que les silhouettes que nous venons d'évoquer. Tout se passe comme si le monstre multiforme et anonyme qu'est «la Communarde» s'apprivoisait dès qu'il a un nom et un visage. Maddalena par exemple, qui pourtant a bien des défauts! Etrangère bien sûr, croqueuse d'hommes, elle berne le faible Blacourt avec la complicité de son amant, l'officier communard Malonsky (encore un métèque), et à la fin du roman se révèle être une espionne prussienne:

1 Lemétreur et Plock sont des personnages du Naufrage de l'Inflexible (Coppée); "les Barbes», de Toute une Jeunesse.

2 Un personnage de bourgeois défenseur de la propriété et participant à l'insurrection est invraisemblable: pourtant il existe, c'est Blacourt de La Commune. Mais ses motivations expliquent cette contradiction (cf. infra).

- Paul et Victor Margueritte, La Commune, Paris, Plon-Nourrit, 1904, pp. 82-83.

Ibid., p. 74.

s Daudet, Contes du Lundi.

- La Commune. 
un comble pour une héroïne de cette révolution patriotique que fut la Commune. ${ }^{1}$

Les motivations de tous ces personnages apparaissent déjà plus ou moins nettement dans l'analyse de leur statut social: l'artisan qui a fait de mauvaises affaires, le jeune bourgeois déclassé et aigri, mais aussi le Parisien qui a "l'émeute dans le sang" ${ }^{2}$ comme l'étranger qui a tout à gagner au désordre, tous attendent de la Commune une revanche à leurs défaites personnelles, la compensation d'un échec dû à leur incurie (Servien fait des vers au lieu de préparer sa licence, Clément boit et politicaille au lieu de s'occuper de son atelier). Dans tous les cas, ces motivations sont individuelles: pas le moindre embryon d'une revendication qui pourrait, fût-ce en pointillé, devenir collective. Là encore, on reconnaît les stéréotypes de la presse et de la littérature versaillaises dans les mois qui suivent immédiatement la Commune: le thème du "fruit sec», ${ }^{3}$ assez bien illustré par Montégut ${ }^{4}$ :

«D'abord les promoteurs, les chefs, les ambitieux: des hommes haineux, des fruits secs, de toute profession, tarés pour la plupart, intelligents peut-être, ce qui double leur faute... Ils voulaient jouir et commander [...] Le mot d'ordre leur était donné par les sociétés secrètes [...] par l'Internationale, par les prophètes du dieu Blanqui, par tous les clubs socialistes, révolutionnaires, ces parlements de prolétaires, comme dit Liebknecht [...] Avec des mots, ils entraînaient le peuple, cet éternel enfant,... mais entre eux ils raillaient la simplicité de ce même peuple qu'ils méprisaient. Aux dernières heures, ils surent disparaître, ceux-là ..... ${ }^{5}$

Tout y est: motivation impure (le Communard est un raté), pas de véritable pensée politique (ils prennent leurs ordres des sociétés secrètes), mépris pour le peuple qu'ils mènent par leur parole (démago-

1 Maddalena sera violée par nombre de Fédérés dans une des dernières scènes du roman: la récurrence de ce genre de scène en fait presque un "passage obligé". De Mme Henry (Une Idylle...) nous ne saurons jamais avec certitude le rôle qu'elle a pu jouer sous la Commune, ce ne sont qu'hypothèses de son ancien amant ( $\mathrm{p}$. 50) ou de Gabriel ( $\mathrm{p} .48$ ) mais ces hypothèses sont étayées par la veulerie opportuniste du personnage, bonapartiste jusqu'à Sedan, qui sans doute a "remplacé le brillant lieutenant de mobiles par quelque colonel fédéré, aux galons irrésistibles" (p. 48) ce qui ne l'empêchera pas, après la répression, d'afficher une liaison élégante et de revenir au monde versaillais.

2 Les Trois Sommations, dans Contes du Lundi.

3 Expression de Richepin et de Du Camp, entre autres.

4 P. Lidsky donne d'autres citations illustrant la même idée, op. cit., pp. 57-61.

- Maurice Montégut, Le Mur, Paris, Dentu, 1892, pp. 17-21. Ce passage, vingt ans après la Commune, n'apporte rien de nouveau par rapport à ce qu'écrit un Du Camp. 
gie) et enfin lâcheté (ils ne savent même pas mourir, ils laissent massacrer le peuple qui les a suivis).

Les frères Margueritte, dont le roman, plus tardif que la plupart, se fait parfois l'anthologie de ces stéréotypes, ont créé, parmi d'autres, deux personnages de Communards caractéristiques: Blacourt, lâche et veule, s'enrôle dans la Commune contre ses propres intérêts, pour tenter de conquérir Maddalena:

"Aller se battre pour ces gueux-là, lui à qui chaque coup de canon était un délice, évoquait Malonsky broyé, - se battre, lui, Blacourt, contre les soldats de l'ordre et de la propriété, contre lui-même, cette démence, à certaines minutes, l'étourdissait comme une fureur de vin, un maléfice... Risquer tout, crever, mais l'avoir.»"

La fin de Blacourt sera aussi dérisoire que sa vie: il est tué par les fédérés qui veulent violer Maddalena, et meurt en voyant sa bienaimée en proie à leur brutalité. ${ }^{2}$ Quant à Malonsky, qui se recommande d'abord par son intrépidité, et qui, lorsque tout est perdu, empêche Maddalena et Blacourt de fuir, ce n'est pas non plus un Communard convaincu, c'est un nihiliste, dont on découvre à la fin qu'il est dément. ${ }^{3}$ Donc, ou le Communard est un lâche, ou, s'il est brave, il est fou.

Il arrive toutefois que le romancier anti-communard peigne des personnages de Communards sympathiques ou touchants: Agricol Mallet, ${ }^{4}$ les Simon, Catisse, ${ }^{5}$ Sorieul, ${ }^{6}$ Jacques Maillandru, ${ }^{7}$ le Turco ${ }^{8}$ etc. Dans ce cas, ce sont des êtres fourvoyés, mêlés à la Commune involontairement, presque à leur insu (Catisse, le Turco), ou, s'ils y prennent une part plus active, dupes de leur naïveté (Mallet, Sorieul, Maillandru). La seule exception serait Simon et sa famille, constellation de générations également en lutte contre l'oppression, seuls à savoir pourquoi ils se battent. Certes, Simon est patriote - il déplore la chute de la colonne Vendôme "sous les yeux des Prussiens" -, mais les Communards ne l'étaient-ils pas? On peut reprocher à Simon de n'être pas, comme Urbaine ou Malavaux cadet, ${ }^{9}$ fidèle à des positions de classe, mais cette carence même est réaliste et objective. Mais les auteurs ferment tout espoir pour Simon: la famille entière est victime

1 La Commune, op. cit., p. 470.

2 Ibid., pp. 583-584.

3 Ibid,, p. 584.

4 Coppée, Euvres Posthumes.

5 La Commune.

- Thérèse.

7 Le Mur.

8 Le Turco de la Commune dans Contes du Lundi.

- Philémon, Vieux de la Vieille. 
de la répression, et Thérèse, seule à survivre, se suicide, sans qu'apparaisse aucun symbole d'avenir comme l'était le nouveau-né dans I.N.R.I. C'est là, et dans la fin du roman, qui cite Hugo: «Oh! patrie, oh! concorde entre les citoyens!», ${ }^{1}$ que se dévoile l'option "conciliatrice», c'est-à-dire anti-communarde, des auteurs. Il reste qu'ils ont crée un personnage d'ouvrier parisien et communard qui dépasse les habituelles caricatures de ce genre de roman.

Indiquons pour finir que, du statut social du personnage et de ses motivations, lesquelles en découlaient, dérive logiquement l'attitude du personnage dans la Commune et après la Commune. Le mauvais ouvrier et le bohême seront des profiteurs: Chouteau, Anatole, ${ }^{2}$ Tudesco. ${ }^{3}$ Même des personnages moins ignobles connaissent leurs instants d'égarement: pour Maillandru, la Commune est aussi l'occasion d'une aventure adultère avec Françoise. Mais plus nombreuses seront les victimes: le roman anti-communard obéit ici à un but de réconciliation nationale. Il ne faut pas hypnotiser le peuple sur sa culpabilité, mais le ramener à la raison en lui montrant qu'il a été égaré par une minorité de furieux, lesquels lui ont laissé payer les pots cassés, mais que luimême a gardé jusque dans sa folie des qualités humaines, qu'il est plus à plaindre qu'à blâmer, bref qu'il est récupérable. Le degré de responsabilité de toutes ces victimes est divers, mais en général assez faible. Le coupable, c'est un être de raison, une entité, "la politique»: "ce naïf poète avait commandé une bande d'ivrognes incendiaires et s'était fait tuer pour une loque rouge! N'était-ce pas révoltant? Oh! l'infamie, la bêtise des rages politiques!» ${ }^{4}$ Les personnages de victimes ne seront pas statiques: ils évolueront de la Commune à un repentir, suicidaire ou constructif, pour leurs fautes. Ils mourront ou non, mais ce sera, plus ou moins, «réconciliés».

Le personnage du roman anti-communard semble donc bien être le lieu privilégié de l'idéologie: beaucoup plus que dans le traitement du récit proprement dit, c'est dans la construction des figures que se révèle le mieux l'intention politique du texte. Nous disons "construction» et non "création» parce qu'il nous a semblé qu'en règle générale, le roman anti-communard ne "génère» pas ses types, mais se contente de les puiser dans le bien commun de la presse et des écrits versaillais, puis de les orner de quelques idiosyncrasies qui ne suffisent pas à les

1 Frères Margueritte, op. cit., p. 638.

2 Dans Thérèse, Anatole et son complice, le criminel Le Fourrier, profitent de la Commune pour faire arrêter Edouard comme Versaillais, et tentent de le fusiller pour s'emparer de sa fortune.

3 Dans Jean Servien, Tudesco, vieil adolescent raté, trouve dans la Commune l'argent et la puissance qui lui ont manqué toute sa vie.

4 Coppée, Guvres Posthumes, Lemerre, tome III, p. 291. 
détacher du stéréotype. Mais en ira-t-il autrement du roman procommunard?

L'étude des personnages dans le roman pro-communard pose un problème spécifique: les romans à envisager sont échelonnés sur un siècle, jusqu'au Canon Fraternité (1970), alors que dans le cas précédent ils l'étaient sur une trentaine d'années seulement. Or en cent ans, les techniques de caractérisation romanesque des personnages ont beaucoup évolué, surtout depuis 1950: il paraît donc difficile, a priori, de traiter de la même manière Urbaine et Marthe, Rabouille ou Florent.

Pourtant, nous serons amenés à constater que, lorsque le romancier choisit la fable $\mathbf{1}^{1}$ le la Commune, fût-ce avec l'intention la plus favorable, les personnages que sécrète cette fable ne semblent guère atteints par l'évolution générale du roman: ils appartiennent plus à l'univers mental du XIX ${ }^{\mathrm{e}}$ siècle romanesque qu'à celui où ils sont effectivement "écrits». Chabrol pourrait échapper à cette objection, puisqu'il fait intervenir dans sa fable deux grands motifs du XXe siècle (la guerre de 1914-18 et la Commune de Berlin, d'une part, la guerre d'Espagne d'autre part), reliant ainsi la Commune de Paris aux révolutions modernes, et se donnant par là même la possibilité d'une écriture qui ne serait pas anachronique. Mais peut-être n'a-t-il pas été jusqu'au bout de cette "mise à jour»: le roman, n'atteignant pas la seconde guerre mondiale et ses suites, ne peut s'écrire comme un roman postérieur à 1950, c'est-à-dire postérieur à Tropismes (Nathalie Sarraute), au Bavard (Louis-René des Forêts) ou à Molloy (Samuel Beckett). Le choix d'une fable centenaire dicte-t-il une écriture aussi dépassée? Ce serait donner sans doute trop d'importance à la fable, mais si nous ne sommes pas en mesure de répondre, nous avouons notre trouble devant ce dilemme qui se pose à la littérature révolutionnaire.

Les personnages favorables à la Commune sont plus nombreux, et plus longuement caractérisés, que les anti-communards. Nous retrouvons la même constatation que pour le roman versaillais, mais il s'agit d'un phénomène inverse: ici, on multiplie les héros positifs, alors que dans le premier cas, on multipliait les caricatures pour accabler l'adversaire. Mais ce fait ne s'explique pas uniquement par l'intention politique. Il obéit d'abord à une remarque de bon sens: la plupart de ces romans sont situés à Paris, et l'adversaire est ailleurs, il n'est donc pas légitime de le montrer. De plus, cet adversaire est souvent anonyme. Ainsi dans I.N.R.I., si on excepte quelques personnages historiques

1 Nous empruntons ce mot à la terminologie des formalistes russes: la fable (par opposition au sujet, qui est le texte dans son déroulement) est l'événement raconté par le livre, mais dans sa chronologie réelle et non textuelle. 
comme Thiers ou les généraux, on chercherait en vain une figure de Versaillais. L'officier qui commande la crucifixion de Râtâs, le noceur versaillais qui charbonne les lettres "I.N.R.I." sur le gibet, sont des ombres, des "utilités". Contrairement à ce qui se passe dans Bas les Cours! (Georges Darien), qui est à mettre un peu à part, il n'y a ici aucune intention de satire sociale: les ennemis sont les instruments du martyre de la Commune, et c'est tout, ils sont réduits à leur fonction, il est inutile de les douer d'une psychologie, d'un nom, d'une origine sociale, etc.

Le roman communard montre rarement les grands adversaires, que ce soit sous forme historique (Thiers) ou sous forme figurée: il s'en prend globalement à la bourgeoisie, à l'armée, parfois à tel ou tel élément du peuple qui, pour diverses raisons, n'est pas favorable à la Commune: ce sont alors les figures marginales du cipal ou du concierge, celui-ci redouté à cause des loyers (le vrai propriétaire n'apparaît guère), ou ce Lumpenproletariat qui se manifeste lorsque des voyous affamés veulent croquer Sans-Mitaines (I.N.R.I.) ou lorsque des charognards repus entonnent parodiquement le Chant des Ouvriers (Canon Fraternité). C'est encore la mère, la fille ou l'épouse du Communard, qui ressent avec crainte et hostilité la Commune où elle ne voit que la destruction de son petit univers (comme la mère Pommier dans L'Apprentie ou Céline dans La Colonne). Quant aux bourgeois, figures caricaturales chez Darien, plus nuancées mais aussi virulentes chez Cassou, ils sont rarement montrés en tant que tels: aucun romancier pro-communard ne saura faire l'équivalent d'un Nucingen ou d'un Leuwen père. Ignorance ou refus? Le personnage de l'anti-communard, dans cette littérature, est trop souvent un marginal. ${ }^{1}$ Parfois même, l'hostilité à la Commune ne se fragmentera en aucun personnage précis: c'est le cas d'I.N.R.I. ou de Philémon.

Les héros positifs, eux, sont nombreux. Leurs auteurs ont eu souci d'éviter, en les décrivant, l'hagiographie, souci que n'avaient guère les auteurs versaillais pour leurs propres héros. Mais y ont-ils réussi?

On décèle en premier lieu, dans le choix des héros, la volonté de montrer la variété sociologique et géographique des combattants de la Commune. Parmi eux se trouvent des ouvriers: Rabouille, Mazoudier, Schramm, ${ }^{2}$ Fournery, les deux Malavaux, Charpin, Gerberoy, ${ }^{3}$ Siffrelin,

1 Le meilleur exemple de cette marginalité nous semble être le personnage de Prophète, l'Invalide de La Colonne, première victime de l'ordre qu'il défend, dupe du mythe de la colonne alors qu'invalide il devrait haïr la guerre, mais qui évoluera sous l'influence de Rabouille.

2 La Colonne.

3 Philémon. 
Flageot, Bizerel, Barbuchet, ${ }^{1}$ mais aussi des bourgeois, des intellectuels: l'instituteur Martin, ${ }^{2}$ Becker, Quiche ${ }^{3}$ et des paysans: Râtâs, Florent. ${ }^{4}$ Cette variété a une grande importance politique: à l'image versaillaise d'une saine France rurale, dressée contre Paris, ville saisie de fièvre, il s'agit d'opposer, non certes une espèce d'union sacrée, mais l'image d'une France où prolétariat rural et prolétariat urbain s'unissent contre la bourgeoisie, aidés en cela par quelques bourgeois transfuges. Dans I.N.R.I. par exemple, cette notion s'élargit jusqu'à devenir presque caricaturale: sur la barricade finale voisinent avec les prolétaires parisiens un marin breton, un rimeur marseillais, un hobereau normand ruiné par l'Empire! Au thème des provinciaux s'ajoute celui des étrangers, dont on sait l'importance dans l'histoire et dans la littérature versaillaises: mais il ne sera le plus souvent traité qu'en effet de réel (Dombrowski, Wroblewski, etc.), Chabrol étant un des rares à avoir forgé des personnages d'étrangers et d'étrangères $d u$ peuple et ralliés à la Commune.

A cette réhabilitation des combattants de la Commune se greffe la réhabilitation de la femme et de l'enfant, par rapport aux figures de la harpie et du voyou du roman et de la presse anti-communards. Philémon cite la fameuse phrase de Dumas sur les «femelles» communardes, et réplique:

«C'est vivantes, citoyen, que les épouses, les mères, les sœurs et les compagnes d'insurgés réhabilitèrent leur sexe dégradé par les chiennes de luxe qui aboyaient aux chausses des prisonniers, le long de l'avenue de Versailles!» ${ }^{5}$

La femme peut n'être qu'une silhouette, comme Ninie Bagarre, ${ }^{6}$ ou une héroïne comme Marie-Rose, ${ }^{7}$ Urbaine, Aurore, ${ }^{8}$ "Baucis»" ou Marthe, cette dernière entourée de toutes les femmes de Belleville qui chargeront de leurs aiguilles et de leurs fuseaux le Canon Fraternité et mourront déchiquetées par l'assaut des Versaillais. Son physique n'est jamais décrit de façon gratuite: sa beauté est toujours révolutionnaire, soit par sa force presque virile (c'est ainsi que le portrait d'Urbaine est

1 Les Massacres de Paris.

2 La Colonne.

3 Les Massacres de Paris.

- Le Canon Fraternité. Deux paysans sont les héros de ces deux romans importants que sont I.N.R.I. et Le Canon.

5 Philémon, op. cit., p. 84.

${ }^{6}$ La Colonne.

7 Les Massacres de Paris.

8 I.N.R.I.

- Philémon. 
celui d'un garçon: avec ses cheveux bruns et sa lèvre duvetée, elle ressemble "trait pour trait à quelque jeune volontaire de l'an II»I), soit par sa fragilité, non aristocratique mais faubourienne, fragilité qui signifie que l'héroïne n'a pas toujours mangé à sa faim: c'est le cas de Marthe. Elles ont dans le roman un rôle souvent capital, puisqu'elles éduquent l'homme qu'elles aiment et le poussent à l'action. ${ }^{2}$

L'enfant, lui, jouera rarement un rôle actif: Nénesse dans La Colonne, Zut-Alors dans I.N.R.I., prennent part à la lutte, mais Adrien dans La Colonne, Jean dans Bas les Cours! ou le bébé d'Urbaine représentent autre chose: l'enjeu de l'avenir, lié au thème de l'éducation, qui sera développé et distordu dans la littérature à l'époque combiste. ${ }^{3}$

Sur le plan des motivations, nous repérons la même volonté de répondre aux calomnies versaillaises. Le héros du roman communard a une histoire, ce n'est pas un amnésique ni un déraciné: il est poussé par une tradition révolutionnaire, il a participé à des complots blanquistes sous l'Empire, comme Rabouille, il est proudhonien, International...4 ou, sans attaches dans un passé révolutionnaire précis, il a reçu une culture humaniste et socialisante qui le prépare à adhérer à la Commune. ${ }^{5}$ Sur cette tradition, sur cette culture, viennent agir parfois un événement, un simple incident, qui créent une véritable metanoia chez le personnage: ainsi Florent, voyant Marthe pauvrement nippée, passera d'un seul coup des "bons principes" d'une révolution vague à un engagement de tout l'être: "depuis tout à l'heure, ce sont mes tripes qui hurlent». ${ }^{6}$ En apparence, cela peut rappeler les motivations troubles (rancœur, misère qui aigrit) décrites par les romanciers anti-communards: en apparence seulement, car l'idée de Chabrol, et avant lui de la plupart des romanciers pro-communards, est qu'on ne devient pas révolutionnaire par une simple décision intellectuelle, mais de tout soi-même.

Si les personnages du roman anti-communard, faiblement ou laide-

1 I.N.R.I., op. cit., p. 24.

2 Nous avons étudié le rôle d'Urbaine plus en détail dans une communication sur Cladel au colloque de la Revue d'Histoire Littéraire de la France sur la Commune.

3 Pour tous ces personnages, et d'autres, nous renvoyons à l'étude de MarieFrance Giaccobi, Influence de la Commune sur le genre romanesque - Les personnages, Diplôme d'Etudes Supérieures (dactylographié), Aix-en-Provence 1970.

4 Descaves dans Philémon a condensé les principales tendances du mouvement ouvrier à l'époque, en y adjoignant un personnage de syndicaliste révolutionnaire moderne, Malavaux cadet, qui critique ses aînés, les met en fureur, et sert à montrer l'inadéquation des positions politiques de la plupart des Communards.

5 Florent.

- Le Canon Fraternité, op. cit., p. 239. 
ment motivés, n'hésitaient pas à changer de camp ou à se repentir, et en tout cas traversaient la Commune en épaves, le héros positif, ici, n'est pas ballotté par les événements, n'est ni victime ni dupe. Il est acteur. Il croise ou non les personnages historiques (Urbaine fusille Thomas, Marthe cache Flourens), mais lui-même est croisé à tout moment par l'histoire.

On a pu leur reprocher leur perfection, ${ }^{1}$ leur langage apprêté, invraisemblable, leurs prônes. ${ }^{2}$ Tout cela est vrai. Mais est-ce le reproche essentiel? Il faudrait ici examiner pour qui ont écrit ces auteurs, et s'ils ont atteint ou non le public qu'ils souhaitaient, ou un public tout court. Tous ont pensé, sur ce problème particulier, que le maintien du personnage était nécessaire pour qu'un roman soit accessible: mais même à ce prix, ces romans sont-ils encore lisibles en 1971? Et pour qui? «Matériellement, un ouvrier n'est pas capable de lire Le Canon Fraternité, et s'il essayait il rencontrerait des mots rares, des allusions mythologiques, historiques et artistiques, qui sûrement échappent à sa compréhension. $\|^{3}$ Le romancier perdrait alors sur les deux tableaux: il aurait sacrifié les audaces formelles et aurait usé de stéréotypes, pour être entendu, donc faire œuvre de messager révolutionnaire; mais s'il n'est pas entendu? Il serait nécessaire de compléter ces questions à peine effleurées ici par une enquête sur un public moderne (les témoignages d'époque nous échappant en grande partie), afin de mesurer l'impact des romans sur la Commune, anciens ou récents.

Au terme de ce rapide survol des romans sur la Commune, avouons un repentir de construction. Nous pensions en commençant, sans trop le dire, pouvoir démontrer que s'appliquait à toutes ces œuvres l'idée de la "Révolution marginale" que nous avions tenté de mettre en lumière avec le symbole du "Chiffre effacé» dans le roman de Cladel. ${ }^{4}$ Or, on ne peut pas affirmer absolument que la Révolution, dans tous ces romans, soit également un «chiffre effacé». Mais si nous n'avons pu le démontrer, nous n'avons pu nous persuader du contraire. La littérature communarde, en dépit de l'adhésion sentimentale qu'elle continue à emporter chez nous, ne nous paraît pas, à beaucoup près, moins «caduque» que la

1 E. Schulkind, op. cit., p. 235.

2 Ibid., id.

3 Palmiro Armando, op. cit., p. 103

4 De l'anecdote du chiffre effacé, sans grande importance en elle-même dans I.N.R.I., nous avons essayé de déduire toute une symbolique, d'où ressort notamment que, chez Cladel, la révolution n'est qu'un chiffre effacé, doublement effacé, sur le papier du livre et dans la trame de l'histoire (Communication au colloque sur la Commune organisé par la Revue d'Histoire Littéraire de la France en novembre 1970). 
littérature adverse. Si on excepte quelques grandes œuvres comme L'Insurgé ou notre cher Philémon, cette littérature, agréable à lire ou non, nous paraît distiller dans son ensemble un ronron rassurant tout aussi pernicieux que celui "d'en face». C'est pour nous défendre contre cette image inadéquate de la Révolution que nous avons tenté, tant bien que mal, de raccorder cette étude à des préoccupations formelles souvent absentes des romans envisagés: raccordement arbitraire peut-être? Mais qui écrit la Révolution? Celui qui parle autour, ou celui qui, en parlant peut-être de tout autre chose, essaie de pratiquer une subversion de l'écriture? A cette question rhétorique, qui dicte sa réponse, opposons un doute: ni l'un ni l'autre, peut-être... mais nous ne croyons pas que ce soit le premier. 